Acta Crystallographica Section D

Biological

Crystallography

ISSN 0907-4449

\section{T. D. Fenn ${ }^{\mathrm{a} *}$ and}

M. J. Schnieders ${ }^{b}$

aDepartment of Bioengineering, Stanford University, Stanford, California, USA, and

${ }^{\mathbf{b}}$ Department of Biomedical Engineering,

University of Texas, Austin, Texas, USA

Correspondence e-mail: fenn@stanford.edu

(C) 2011 International Union of Crystallography

Printed in Singapore - all rights reserved

\title{
Polarizable atomic multipole X-ray refinement: weighting schemes for macromolecular diffraction
}

In the past, weighting between the sum of chemical and databased targets in macromolecular crystallographic refinement was based on comparing the gradients or Hessian diagonal terms of the two potential functions. Here, limitations of this scheme are demonstrated, especially in the context of a maximum-likelihood target that is inherently weighted by the model and data errors. In fact, the congruence between the maximum-likelihood target and a chemical potential based on polarizable atomic multipole electrostatics evaluated with Ewald summation has opened the door to a transferable static weight. An optimal static weight is derived from first principles and is demonstrated to be transferable across a broad range of data resolutions in the context of a recent implementation of X-ray crystallographic refinement using the polarizable AMOEBA force field and it is shown that the resulting models are balanced with respect to optimizing both $R_{\text {free }}$ and MolProbity scores. Conversely, the classical automatic weighting scheme is shown to lead to underfitting or overfitting of the data and poor model geometry. The benefits of this approach for low-resolution diffraction data, where the need for prior chemical information is of particular importance, are also highlighted. It is demonstrated that this method is transferable between low- and high-resolution maximumlikelihood-based crystallographic refinement, which proves for the first time that resolution-dependent parameterization of either the weight or the chemical potential is unnecessary.

\section{Introduction}

Crystallographic refinement of atomic coordinates involves optimization against both a chemical potential energy (or force field, $\left.E_{\text {ForceField }}\right)$ and the observed data $E_{\mathrm{X} \text {-ray, }}$

$$
E_{\text {tot }}=E_{\text {ForceField }}+w_{\mathrm{A}} E_{\mathrm{X} \text {-ray }} \text {, }
$$

where $w_{\mathrm{A}}$ weights the data relative to the force-field component. We assume throughout that weighting of other parameters, such as occupancies and $B$ factors, is independent of coordinates. Also, we do not consider the case of additional experimental information such as neutron or real-space data, although much of what is discussed below is also applicable to these cases (see, for example, Fenn et al., 2011). A widely adopted standard for determining an optimal $w_{\mathrm{A}}$ involves calculating the ratio of the gradient norms between the two objective functions,
Received 11 July 2011

Accepted 22 September 2011 


$$
w_{\mathrm{A}}=\frac{\left\|\nabla E_{\text {ForceField }}\right\|}{\left\|\nabla E_{\mathrm{X} \text {-ray }}\right\|}
$$

as originally described by Brünger (1988) and standardized over the course of several years (Wang \& Stubbs, 1993; Adams et al., 1997). Therefore, if the X-ray gradient is smaller than the chemical gradient on average, it is upweighted to balance the two potentials and vice versa (Fig. 1).

The standard formalism for the $E_{\mathrm{X} \text {-ray }}$ term in (1) as it is applied to macromolecular structures utilizes a likelihood target (hereafter referred to as 'crystallographic likelihood') that includes both data and model error. The latter is accounted for by inflating the total variance and downweighting the model contribution owing to incompleteness and coordinate error [the full details of the likelihood target will not be discussed here; the reader is referred to McCoy (2004) and references therein for a more thorough discussion], causing the likelihood to become less peaked and broader as the model error increases. A similar method of weighting the data is common in model refinement in the context of NMR (Habeck et al., 2006).

The crystallographic likelihood can be stated as the probability of observing the measured data $(D)$ given an atomic model $(X)$. This can be formally written as a conditional probability $p(D \mid X)$. However, what we seek to optimize is the probability of the atomic model given the measured data, $p(X \mid D)$. This is accomplished through Bayes' rule,

$$
p(X \mid D) \propto p(D \mid X) p(X),
$$

where $p(X)$ represents the prior probability of the atomic model based on our established understanding of molecular energetics. Although computational solutions to the Schrödinger equation (i.e. quantum mechanics) are the gold standard for molecular energetics, $a b$ initio electronic structure calculations that use a reliable basis set and account for electron correlation are prohibitively expensive for macromolecular refinement. For this reason, a modern polarizable force field, such as AMOEBA, that accurately reproduces the quantum-mechanical potential energy surface is the most compelling alternative for prior chemical information (Ponder et al., 2010; Ren et al., 2011).
The relative probability of an atomic conformation is given by its Boltzmann factor $\exp [-U(X) / k T]$ and therefore (3) can be expressed as

$$
p(X \mid D) \propto p(D \mid X) \exp [-U(X) / k T],
$$

where $U(X)$ is the potential energy of the crystal according to a polarizable force field. For convenience, the maximum of $p(X \mid D)$ is determined by minimization of its negative logarithm, which yields a hybrid energy function for the model $E(X)$,

$$
E(X)=-\log [p(D \mid X)]+U(X) / k T .
$$

This is identical to the form presented initially in (1) and identifies $w_{\mathrm{A}}=k T$ as the expected value of the weight to apply to the crystallographic likelihood to balance the two terms. At $300 \mathrm{~K}, k T$ equals 0.6 (on a kcal mol ${ }^{-1}$ scale) such that if the prior chemical information is of high quality then weights close to 0.6 are expected to balance model agreement with respect to both the observed data (i.e. $R_{\text {free }}$ ) and prior chemical knowledge (i.e. MolProbity score).

Independent of the target type used, the effect of (2) tends to upweight the weaker (flatter) potential, which may not be desirable if the weaker potential is associated with low confidence. To illustrate this, in the extreme case of subatomic resolution (>1.0 $⿱$ ) data, the crystallographic likelihood and its derivatives will be sharply defined (illustrated by the red curve in Fig. 1c). The effect of (2), in this case, will be to downweight the data (indicated by the transition from Fig. $1 c$ to Fig. $1 b$ ). If the potential wells are not congruous, this will cause the coordinates to move away from the well determined data-based target and towards the chemical prior potential 'well'. Likewise, in the opposing extreme of poor data, the crystallographic likelihood will be relatively flat (red curve in Fig. 1a). (2) will have the effect of upweighting the data in this case, causing the atoms to move away from the chemical prior 'well' and towards the uncertain X-ray potential 'well' (indicated in Fig. 1 by the transition from Fig. $1 a$ to Fig. 1b). The resulting effect of this scheme will cause low-resolution data sets to be overweighted, yielding models with poor geometry and potentially increased $R_{\text {free }}$ values owing to overfitting. Conversely, high-resolution data sets will be downweighted, leading to inflated $R$ and $R_{\text {free }}$ values and models with
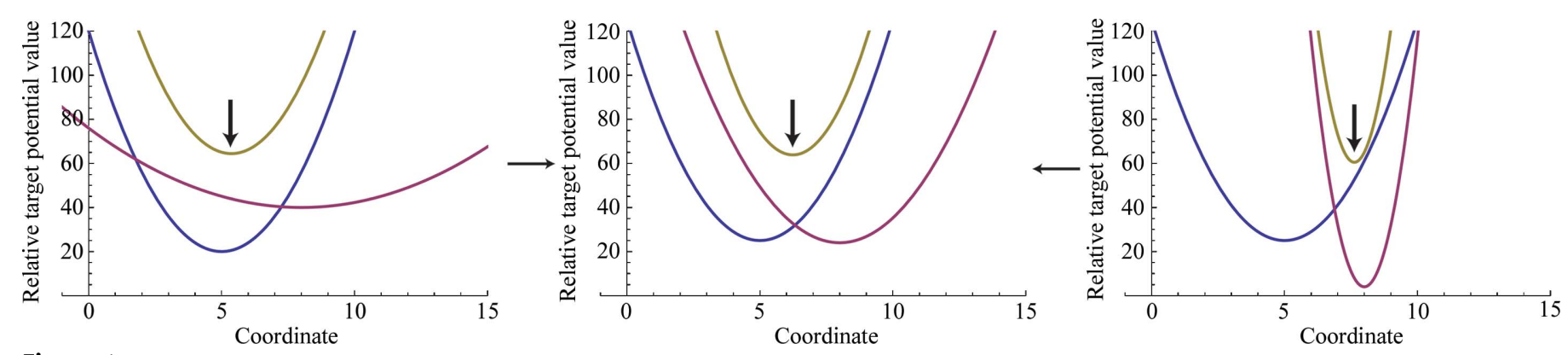

Figure 1

The effect of no weighting on X-ray (red) and chemical (blue) potentials in noncongruous cases, with the total potential shown in yellow. The global minimum is marked by a thick downward-pointing arrow in each case. In the case of low-resolution or poorly measured X-ray data $(a)$, the X-ray potential will be weakly defined and the minima will favor the chemical potential. Alternatively, in the case of well measured or high-resolution X-ray data $(c)$, the X-ray potential will be sharp and cause the minima to favor the data. The effect of equation (2) is to bring these two potentials onto the same scale as the chemical potential $(b)$. 
conservative geometry. This may partly explain the noted correlation between data resolution and measures of model quality (Arendall et al., 2005; Bhattacharya et al., 2007). While options exist to test a variety of $w_{\mathrm{A}}$ values, the default behavior in most likelihood-based refinement packages is to utilize the mechanism in (2), which may partly be a consequence of limitations in the force fields commonly used in crystallographic refinement, as also suggested by McCoy (2004).

Ideally, the force field and data-based target will be congruent: i.e. the minima for the two objective functions describing these terms are equivalent and the weighting between them is therefore less critical. It is reasonable to hypothesize that the degree of overlap/agreement should correlate with the level of detail used in describing the molecular physics of the chemical system. For example, models that take into account charge interactions of ions with their full environment (including long-range interactions), polarization, bond-angle coupling effects etc. will more accurately reproduce the atomic forces within the crystal. Our previous work illustrated an improved congruence between the polarizable AMOEBA force field and the crystallographic target (Schnieders et al., 2011) with less dependence on the weighting scheme for a variety of resolutions and data qualities. Further, the benefit of refining against higher quality biomolecular physics (i.e. a polarizable atomic multipole force field) has been shown in several X-ray and neutron crystallographic contexts (Schnieders et al., 2009; Fenn, Schnieders, Brunger et al., 2010; Fenn et al., 2011). However, it is important to point out that even the most modern approaches fail to recognize that crystal conformations are governed by free energy rather than potential energy. Optimization based on a potential energy is equivalent to quenching the crystal to $0 \mathrm{~K}$. Future work is needed to incorporate entropic effects into the prior probability of the model during crystallographic refinement, similar to efforts made in the protein-folding field (Faver et al., 2011).

Here, we have sought to more extensively test the effect of weighting on the resultant $R$ values and model geometry using our recently developed molecular-physics platform called Force Field $X(F F X)$ that executes within the Java Runtime Environment (JRE). This includes an implementation of the atomic multipole optimized energetics for biomolecular applications (AMOEBA) force field (Ren \& Ponder, 2003, 2004; Ponder et al., 2010; Ren et al., 2011), a particle mesh Ewald (PME) method (Darden et al., 1993; Essmann et al., 1995; Sagui et al., 2004) using shared memory parallelization and symmetry operators for efficient computation of electrostatic energies (Schnieders et al., 2011) and a crystallographic likelihood target using a differentiable bulk-solvent model (Fenn, Schnieders \& Brunger, 2010). By re-refining 18 macromolecular models spanning a range of resolutions, we show the agreement between the chemical and crystallographic potentials achieves a good balance - and is typically optimized in both cases - by scaling the X-ray component using a $w_{\mathrm{A}}$ value close to $k T$. In effect, the maximumlikelihood target and prior chemical information are both in units of $\mathrm{kcal} \mathrm{mol}^{-1}$, which has the advantage of aiding chemical interpretation of model improvements. This suggests rigorous molecular physics coupled with crystallographic likelihood does not require resolution-dependent or dataquality-dependent weighting schemes. We also illustrate the effects of automatic weighting and its potential pitfalls.

\section{Methods}

Testing was performed on 18 structures selected from the PDB to encompass a variety of starting model qualities as determined from MolProbity scores (Chen et al., 2010) and a broad resolution range. The PDB entries selected based on these criteria were $3 \mathrm{bbw}, 3 \mathrm{crw}, 2 \mathrm{r} 4 \mathrm{r}, 1 \mathrm{a} 7 \mathrm{~b}, 1 \mathrm{bl}$, 3ffn, 2quk, 2fnp, 3g8l, 1sfc, 3et9, 2r0f, 3gpn, 1mhq, 3o7h, 1dp0, 2ipr and 2rk4.

Models were prepared by using the CNS generate.inp script, which carries out a brief Cartesian dynamics step (50 steps of 5 fs each) and adds $\mathrm{H}$ atoms if necessary to the input coordinates. The model generated in this fashion was then used as input for one of four different refinement methodologies. The first involved using an OPLS-AA-X force field with electrostatics computed using a spherical cutoff of $8.5 \AA$ (with a switching function that begins taking effect at $6.5 \AA$ ) and the CHARM22 version of the TIP3P water model as implemented in $C N S$ v.1.3 (Jorgensen \& Tirado-Rives, 1988; Engh \& Huber, 1991; Linge et al., 2003; Nozinovic et al., 2010; Schröder et al., 2010; Fenn et al., 2011). The weight $w_{\mathrm{A}}$ was set to either 1.0 or left to the automatic setting as per (2). Only local optimization was performed based on three macrocyles of refinement which included 150 coordinate and $100 \mathrm{~B}$-factor minimization steps. The second and third refinement methodologies utilized PHENIX v.1.6.4 and REFMAC v.5.0109, respectively, which include an Engh and Huber restraint set based on the CIF dictionary model (Engh \& Huber, 1991; Vagin et al., 2004; Adams et al., 2010) and no treatment of electrostatics. For the PHENIX refinements, three macrocycles of 150 maximum iterations were performed and weights were fixed at 1.0 or determined automatically. For the REFMAC-based refinements, 50 cycles of combined coordinate and $B$-factor optimization were performed using automatic weighting only (using the 'weight auto' option). An appropriate static weight for each model was not determined for the REFMAC refinements, as there is no standard method for its determination. None of the refinement methods employed additional restraints (e.g. hydrogen bond, NCS, Ramachandran restraints etc.) other than the default parameterizations encoded in the programs.

The fourth refinement method involved our recently described $F F X$ platform (Fenn, Schnieders \& Brunger, 2010; Schnieders et al., 2011). The chemistry term in $F F X$ is based on the AMOEBA force field (Ren \& Ponder, 2003, 2004; Ponder et al., 2010; Ren et al., 2011) with electrostatics computed using a symmetrized particle-mesh Ewald approach (Sagui et al., 2004; Schnieders et al., 2011). The X-ray term is based on the maximum-likelihood model determined using spline coefficients (Murshudov et al., 1997; Cowtan, 2002, 2005) and the methodology of Fenn for derivatives with respect to atomic coordinates and bulk solvent (Brünger, 1989; Fenn, 
Schnieders \& Brunger, 2010). The bulk solvent itself was modeled using a differentiable polynomial switch model (Fenn, Schnieders \& Brunger, 2010). Electron density for the atomic model was computed using the isolated-atom formalism as described by Schnieders et al. (2009). Structure factors for both terms were computed using fast Fourier methods (Ten Eyck, 1973, 1977) and symmetry was applied in reciprocal space if necessary (Waser, 1955; Brünger, 1989) using the recommendations of Bricogne for sampling and symmetrization of structure factors (Bricogne, 2006). Fitting of scale factors between the model and data structure-factor amplitudes are based on the methods of Afonine et al. (2005b). Local optimizations were performed to an r.m.s. gradient convergence of $0.1 \mathrm{kcal} \mathrm{mol}^{-1} \AA^{-1}$ and $B$-factor optimizations were converged to an r.m.s. gradient of 0.01 (unitless).

Following refinement, models were analyzed using MolProbity (Chen et al., 2010) and hydrogen bonds were tabulated and analyzed (e.g. for computation of the percentage of hydrogen bonds; Table 1) using the Hydrogen Bond Analysis Tool (HBAT; Panigrahi \& Desiraju, 2007).
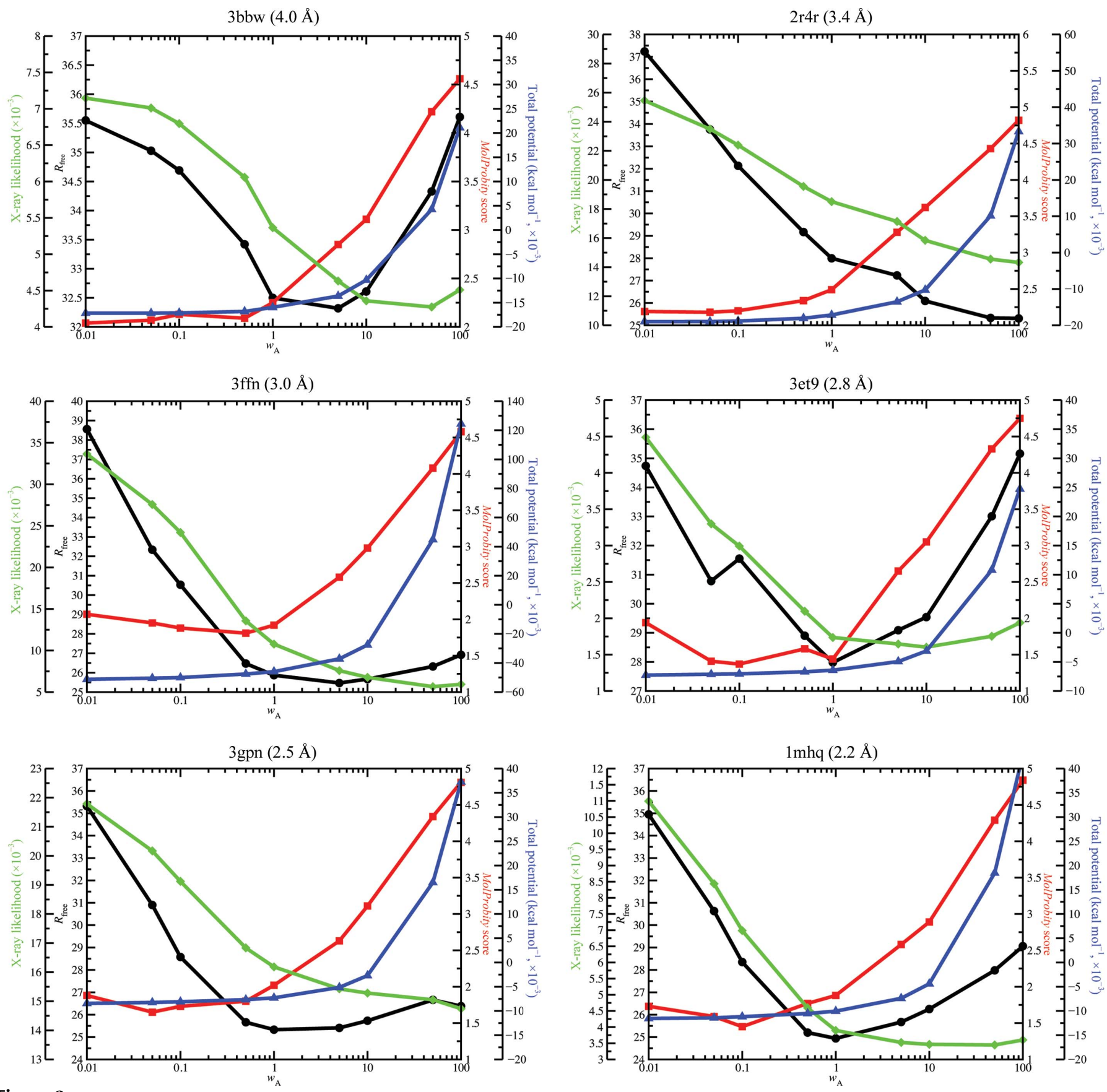

Figure 2

Plot of $R_{\text {free }}$ (left $y$ axis; black), total X-ray potential (second left $y$ axis; green), MolProbity scores (right $y$ axis; red) and total chemical potential (second right $y$ axis; blue) versus $w_{\mathrm{A}}$ (logarithmic scale) for six representative structures ranging from 2.2 to $4.0 \AA$ resolution (PDB codes and resolutions are given above each respective plot). 


\section{Results}

To determine optimum weights for $F F X$, we initially performed a grid search centered around a $w_{\mathrm{A}}$ of 1.0 for 18 different structures spanning diffraction limits of 1.2-4.0 resolution. Representative plots for ten of these structures in terms of $R_{\text {free }}$, MolProbity score and the total chemical and $\mathrm{X}$-ray targets as a function of $w_{\mathrm{A}}$ are illustrated in Figs. 2 and 3. Briefly, the MolProbity score of a model is computed from a combination of geometric factors such as clashscore, percentage of bad rotamers and unfavorable Ramachandran percentage (Chen et al., 2010). The resultant value is intended to reflect an estimate of the data resolution for the model. On average, a $w_{\mathrm{A}}$ value of 1.0 is a good balance between the $R_{\text {free- }}$ and MolProbity-based geometry scores. The $R_{\text {free }}$ did suggest a tendency to favor higher $w_{\mathrm{A}}$ values in the case of lowresolution data $(<2.5 \AA)$, although this coincides with elevated MolProbity scores that may not justify the trade-off. Increasing or decreasing the weight by more than an order of magnitude leads to underfitting or overfitting of the data and conservative or poor geometry scores, respectively. The exception to this trend $\left(2 \mathrm{r} 4 \mathrm{r}\right.$ ) refined to a $2.5 \%$ lowered $R_{\text {free }}$ value using a weight of 100.0. However, this also led to increased overfitting (as monitored by the gap between $R$ and $R_{\text {free, }}$ which more
Table 1

Refinement results for a low-resolution crystal structure (PDB entry $3 \mathrm{crw}$ ) using either the automatic weighting scheme (equation 2 ) or a weight of 1.0 .

MolProbity scores reflect an estimate of the data resolution and percentile scores are given in parentheses. See $\$ 2$ for details of refinements. For a table of results over all structures, see Table 2 .

\begin{tabular}{|c|c|c|c|c|c|c|}
\hline Method & $w_{\mathrm{A}}$ & $\begin{array}{l}R_{\text {free }} \\
(\%)\end{array}$ & $\begin{array}{l}\text { MolProbity } \\
\text { score }\end{array}$ & $\begin{array}{l}\text { R.m.s.d. } \\
\text { bonds }(\AA)\end{array}$ & $\begin{array}{l}\text { R.m.s.d. } \\
\left.\text { angles ( }{ }^{\circ}\right)\end{array}$ & $\begin{array}{l}\% \text { of hydrogen } \\
\text { bonds }\end{array}$ \\
\hline PDB & - & 35.40 & $3.05(86)$ & - & - & - \\
\hline \multirow[t]{2}{*}{ PHENIX } & 4.9 & 39.95 & $4.78(2)$ & 0.027 & 2.68 & 15 \\
\hline & 1.0 & 38.65 & $3.56(63)$ & 0.005 & 0.83 & 17 \\
\hline \multirow[t]{2}{*}{ CNS } & 18.7 & 39.72 & $3.66(57)$ & 0.010 & 0.92 & 25 \\
\hline & 1.0 & 33.60 & $2.83(93)$ & 0.004 & 0.60 & 30 \\
\hline REFMAC & 208.3 & 35.71 & $3.62(59)$ & 0.012 & 1.37 & 18 \\
\hline$F F X$ & 1.0 & 31.06 & $2.20(100)$ & 0.014 & 2.78 & 32 \\
\hline
\end{tabular}

than doubled from $4.7 \%$ to $10.2 \%$; data not shown) and a poor MolProbity score, suggesting that this improvement comes at a significant cost. The insensitivity of the $R_{\text {free }}$ to overfitting in this case may be reflective of poor model and/or data quality and suggests that $R_{\text {free }}$-based optimizations of the $w_{\mathrm{A}}$ (Brünger, 1992) value without considering consequences to model geometry can be misleading in some cases. The trends in $R_{\text {free }}$ are similar to those observed for the free likelihood
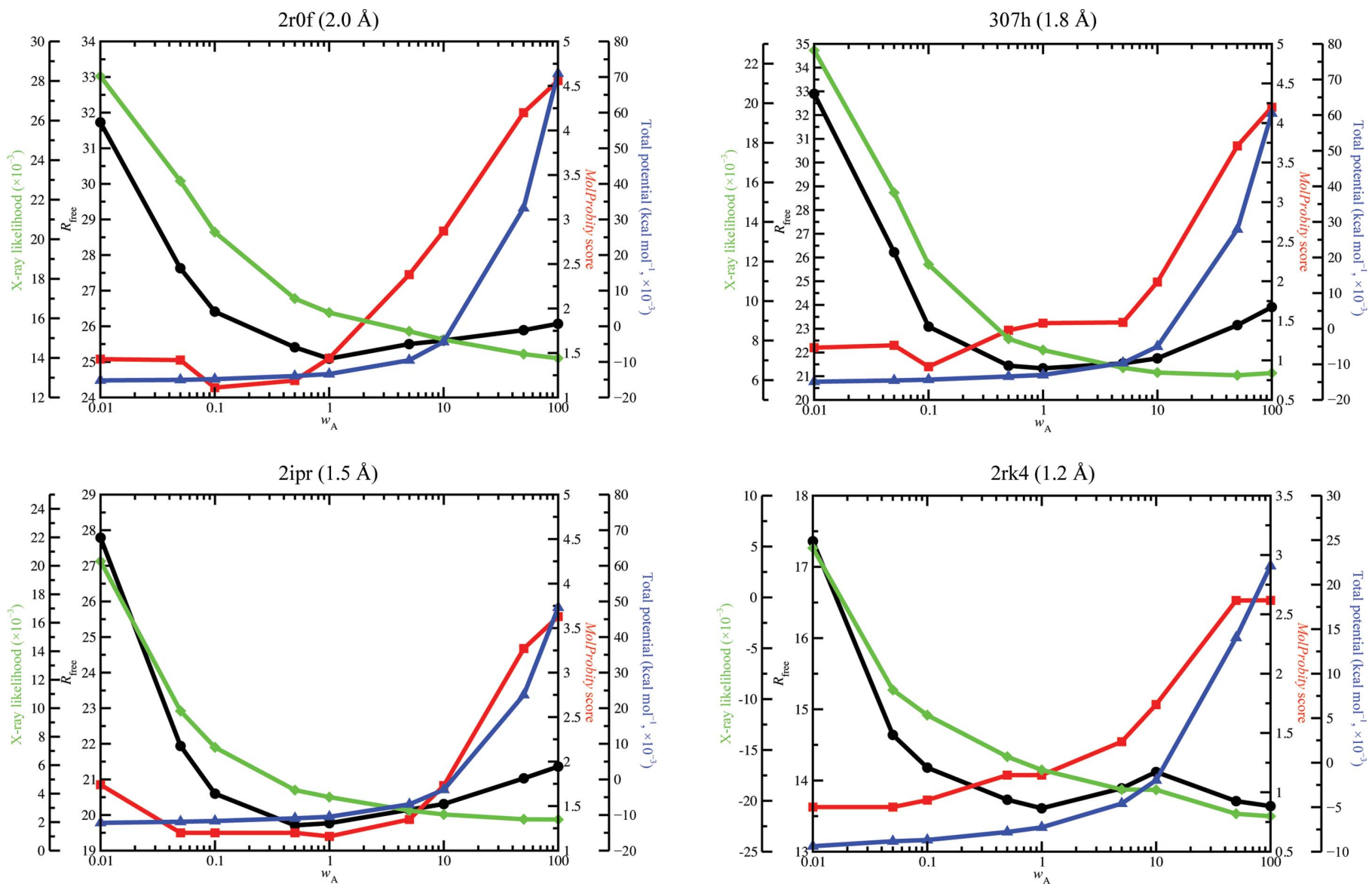

Figure 3

Plot of $R_{\text {free }}$ (left $y$ axis; black), total X-ray potential (second left $y$ axis; green), MolProbity scores (right $y$ axis; red) and total chemical potential (second right $y$ axis; blue) versus $w_{\mathrm{A}}$ (logarithmic scale) for four representative structures ranging from 1.2 to $2.0 \AA$ A resolution (PDB entries and resolutions are given above each respective plot). 
(i.e. the crystallographic likelihood computed for the free set of reflections; see Supplementary Figs. 1 and $2^{\mathbf{1}}$ ), suggesting that the potential effect of 'unweighted' statistics is negligible (Ford \& Rollett, 1970).

The finding that a $w_{\mathrm{A}}$ value of 1.0 is transferable across resolutions and leads to a good balance between model geometry and agreement with the data should extend to other refinement engines that employ maximum-likelihood-based targets. To illustrate this and compare it with the effect of automatic weighting, we refined this group of structures using either the weighting scheme outlined in (2) or using a static weight of 1.0 using the OPLS-AA-X force field as implemented in $C N S$ v.1.3. Following refinement, we compared the resultant MolProbity metrics and differences between $R_{\text {free }}$ and $R$ (as a measure of overfitting) as a function of model resolution and the results are presented in Fig. 4. With lowresolution data (Figs. $4 a$ and $4 c$ ), the automatic weighting scheme leads to systematic increases in MolProbity scores (note the gap between the green diamonds and the blue triangles in Fig. 4c) and overfitting (as illustrated by the gap between the black circles and the red squares in Fig. 4a) versus a static weight of 1.0. At higher resolution (approximately $2.5 \AA$ and higher; Figs. $4 b$ and $4 d$ ), the MolProbity scores using either a static or automatic weight are in close agreement, while the trend in overfitting is reversed (note the inversion in the relationship between the black circles and the red squares in Figs. $4 a$ and $4 b$ and the convergence between the green and blue data points between Figs. $4 c$ and $4 d$ ). This follows the hypothesis presented earlier: high-resolution data sets will lead to systematically low weighting of the data, while at low resolution the trend is reversed, leading to significant overfitting and poor model geometry. This is also apparent in the $w_{\mathrm{A}}$ values themselves (shown in magenta).

In the case of low-resolution data, the crystallographic likelihood target will be relatively flat and contains less information. This leads to a strong dependence on the atomic forces provided by the force field to augment the refinement of models against such diffraction data. Therefore, it is critical that the force field is accurate and transferable for the resultant model to agree with the data and expectations based on our established understanding of chemistry and electrostatics. Although fixed-charge force fields, such as those used in $C N S$, have been tuned over many years, their lack of an explicit treatment of electronic polarization limits their transferability between vacuum and condensed phases (Ponder \& Case, 2003; Lopes et al., 2009). For example, incorporation of electrostatic effects in the force field should lead to stable hydrogen bonding and thereby improve atomic positioning. Such hydrogen bonding defines secondary-structural motifs such as $\beta$-sheets, which provide a test case for this hypothesis. This is illustrated in Fig. 5 for a $4 \AA$ model refined using automatic weighting and Engh and Huber (EH) based restraints (Engh \& Huber, 1991; Vagin et al., 2004) as implemented in PHENIX

\footnotetext{
${ }^{\mathbf{1}}$ Supplementary material has been deposited in the IUCr electronic archive (Reference: DZ5236). Services for accessing this material are described at the back of the journal.
}

Table 2

Average refinement results for 17 crystal structures using either the automatic weighting scheme (equation 2) or a weight of 1.0.

One of the 18 models used in Fig. 4 (2rk4) was omitted owing to the presence of anisotropic temperature factors, which are not available in CNS-based refinements. MolProbity scores reflect an estimate of the data resolution and percentile scores are given in parentheses. See $\$ 2$ for details of refinements.

\begin{tabular}{llllllll}
\hline $\begin{array}{l}\text { Resolution } \\
(\AA)\end{array}$ & Method & $w_{\text {A }}(\%)$ & $\begin{array}{l}R_{\text {free }} \\
(\%)\end{array}$ & $\begin{array}{l}\text { MolProbity } \\
\text { score }\end{array}$ & $\begin{array}{l}\text { R.m.s.d. } \\
\text { bonds }(\AA)\end{array}$ & $\begin{array}{l}\text { R.m.s.d. } \\
\text { angles }\left({ }^{\circ}\right)\end{array}$ \\
\hline 2.68 & PDB & - & 23.58 & 27.65 & $2.95(53)$ & - & - \\
& PHENIX & 7.4 & 21.44 & 29.27 & $3.19(39)$ & 0.022 & 2.20 \\
& & 1.0 & 21.73 & 29.71 & $2.36(80)$ & 0.004 & 0.80 \\
& CNS & 5.6 & 24.07 & 30.06 & $2.70(67)$ & 0.008 & 0.84 \\
& & 1.0 & 26.49 & 29.84 & $2.37(84)$ & 0.005 & 0.63 \\
& & & \\
& REFMAC & 2.3 & 19.55 & 29.76 & $2.54(73)$ & 0.015 & 1.52 \\
& FFX & 1.0 & 21.57 & 27.51 & $1.96(95)$ & 0.013 & 2.61 \\
\hline
\end{tabular}

(Adams et al., 2010; Figs. 5a and 5c) or a $w_{\mathrm{A}}$ of 1.0 and the AMOEBA force field as implemented in $F F X$ (Figs. $5 b$ and $5 d$ ). Protein main-chain atoms resulting from refinements using the EH-based restraints alone (Fig. 5a) are significantly shifted such that hydrogen bonds are weak or not formed at all. For example, the amino acid at the upper leftmost position in Fig. 5(a) rotates out of the $\beta$-sheet plane and is therefore no longer properly positioned to form a hydrogen-bond interaction. These factors negatively influence the resultant MolProbity scores and the percentage of calculated intramolecular hydrogen bonds, and lead to poor agreement with the data regardless of the weighting scheme employed for this test model using EH-based methods (Table 1). These results are consistent with respect to the models selected, as reflected in the averages across the test set (Table 2). Use of a fixedcharge OPLS-AA force field with conditionally convergent electrostatics (as implemented in CNS) shows some improvement over the EH-based results (Table 1). The AMOEBA model as implemented in $F F X$ improves the MolProbity-based effective model resolution by up to $2.5 \AA$ and improves the agreement with the data as indicated by lower $R_{\text {free }}$ values. Also, hydrogen bonds are more regular in terms of distance (i.e. closer to the canonical $1.96 \AA$ separation) and $\mathrm{N}-\mathrm{H}-\mathrm{O}$ angle (approximately $165^{\circ}$, compare Figs. $5 c$ and $5 d$ ) in the $\beta$-sheet structure (Baker \& Hubbard, 1984; Fabiola et al., 1997). The electron density is too weak to explicitly determine the locations of these atoms (Supplementary Fig. $3^{\mathbf{1}}$ ). The angular energetics of hydrogen bonding are properly modeled in the $F F X$ case primarily owing to the polarizable atomic multipole description of the molecular electrostatics. For an extensive comparison of AMOEBA hydrogen-bonding energy, distance and orientational dependence compared with OPLS-AA and MP2/aug-cc-pvTZ electronic calculations, see Ren et al. (2011). These results are in agreement with other tests of the effect of electrostatics on macromolecular refinement (Schnieders et al., 2011; Fenn et al., 2011).

\section{Conclusions}

Evaluation of the X-ray refinement of macromolecular structures is complicated by the lack of a global measure of 
both model quality and agreement with the data. The crystallographer is ultimately responsible for post facto analysis of the resultant model to assess the trade-offs between emphasizing geometry relative to $R / R$ free values and alter, if necessary, the weighting between the chemical and data-based potentials to eventually find an optimum balance. Furthermore, this process is subjective and prone to error and/or misinterpretation. Equation (2) is intended to alleviate this necessity by upweighting the weaker potential; however, this is not desirable in a maximum-likelihood context where the data-based potential is naturally weighted by the model and data uncertainty. We therefore sought to further analyze this problem, particularly in the context of polarizable force fields, where the chemical potential is more accurate and transferable than in the canonical Engh and Huber/CIF-based methods (Engh \& Huber, 1991; Vagin et al., 2004) or fixedcharge force fields (Ponder \& Case, 2003).

The automatic weighting scheme described by (2) led to a resolution-dependent weight that overweights low-resolution diffraction data (i.e. $w_{\mathrm{A}}>1.0$ ), causing increased overfitting

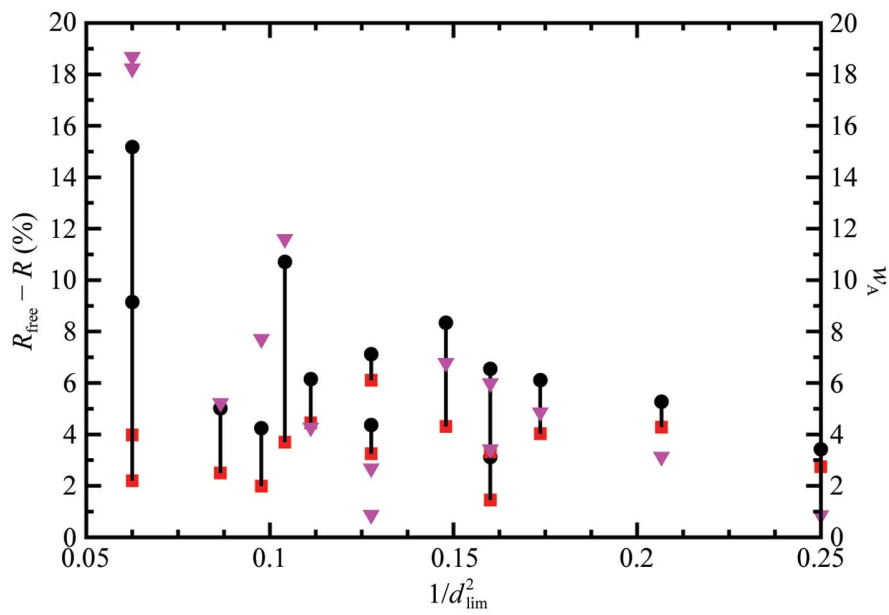

(a)

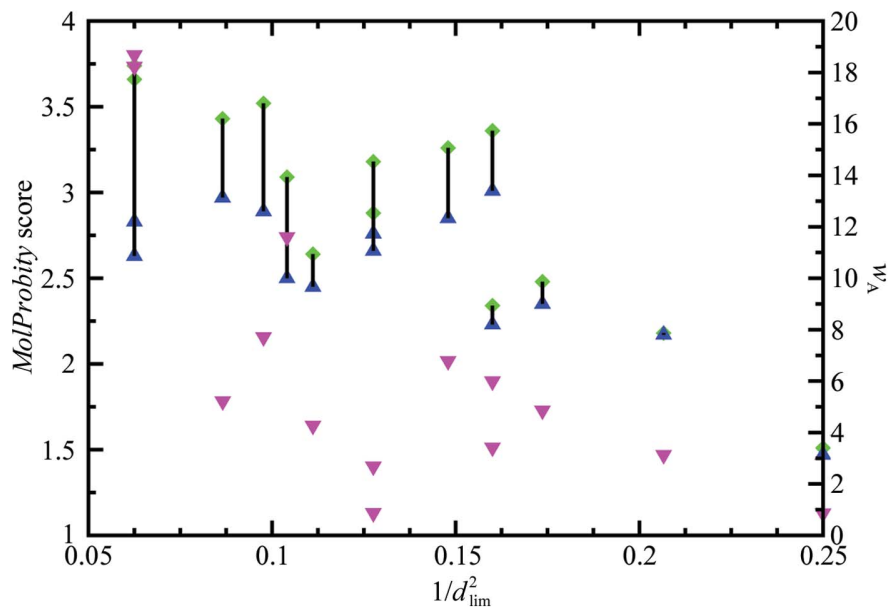

(c) and poor geometry scores (Figs. $4 a$ and $4 c$ ). In the opposing extreme of high-resolution data $(>2.0 \AA)$, the automatic weights led to underfitting (i.e. $\left.w_{\mathrm{A}}<1.0\right)$ coupled with conservative MolProbity scores (Figs. $4 b$ and $4 d$ ). In the case of $F F X$, refinement of a variety of structures ranging from 1.2 to $4.0 \AA$ resolution across a number of $w_{\mathrm{A}}$ values using AMOEBA chemical forces and a likelihood target for the data (see §2) suggest that a $w_{\mathrm{A}}$ of 1.0 tends to yield optimum geometric scores and $R_{\text {free }}$ values (Figs. 2 and 3), which is on the order of our prediction of $k T$ based on Bayes' law. The inherent weighting of the crystallographic data by the likelihood function coupled with a polarizable force field essentially eliminates the need for varying the weight compared with previous-generation refinement methods.

The importance of congruence between the chemical and data-based potentials is particularly problematic with respect to low-resolution diffraction data. In such cases, prior chemical information that does not accurately reproduce atomic forces, such as hydrogen bonding as described by polarizable electrostatics, will lead to poor model quality

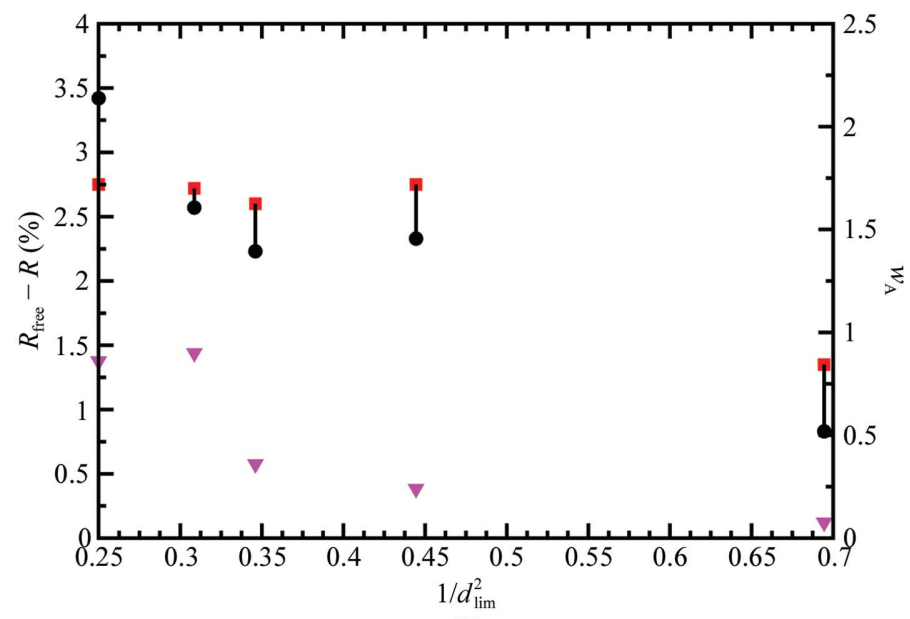

(b)

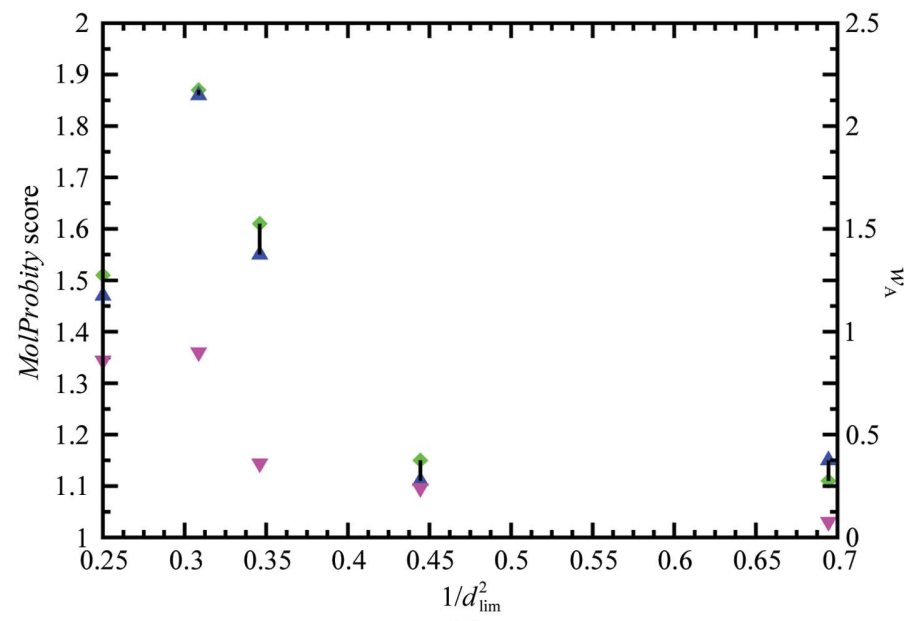

(d)

Figure 4

Plot of $R_{\text {free }}-R$ and MolProbity scores as a function of resolution. Black and red points refer to $R_{\text {free }}-R$ differences $(a$ and $b)$ using either the automatic weight (black circles) or a $w_{\mathrm{A}}$ of 1.0 (red squares), while green and blue points refer to MolProbity scores ( $c$ and $d$ ) using either the automatic weight (green diamonds) or a $w_{\mathrm{A}}$ of 1.0 (blue triangles). Black lines are drawn connecting the automatic weight values with those obtained using a weight of 1.0. The automatically determined $w_{\mathrm{A}}$ values themselves are also shown as magenta triangles. $(a)$ and $(c)$, structures ranging from 2.5 to $4.0 \AA$ resolution. $(b)$ and $(d)$, structures ranging from 1.2 to $2.5 \AA$ resolution. 
because this information is weak or absent in the crystallographic likelihood target. A significant body of methodology has been directed at this problem, including elastic networks (Schroder et al., 2010; Murshudov et al., 2011), conformationally dependent libraries (Berkholz et al., 2009; Tronrud et al., 2010), studies of $\tau$ angles (Touw \& Vriend, 2010) and hydrogen-bond restraints (Fabiola et al., 2002; Echols et al., 2010). Rather than add/reparameterize non-electrostatic based restraints to correct for the neglect of electrostatics or to augment inherently limited fixed-charge electrostatics, we believe the polarizable atomic multipole AMOEBA force field represents a more fundamental solution. The AMOEBA model has been systematically designed to reproduce the

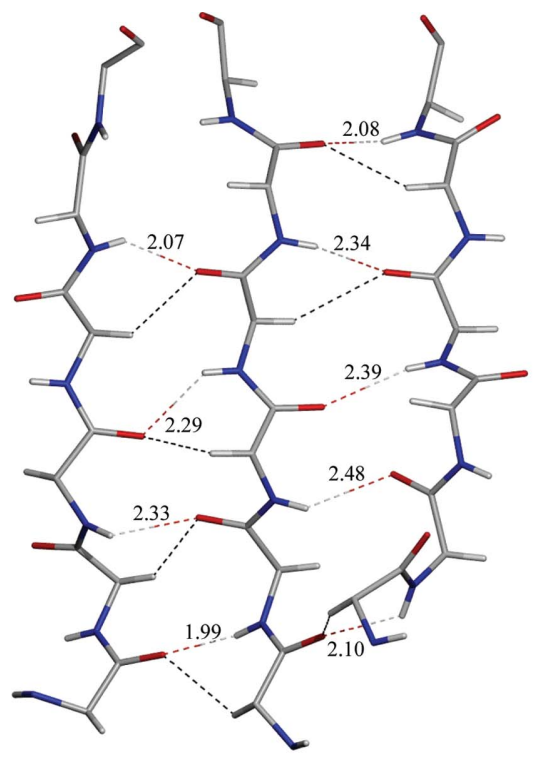

(a)

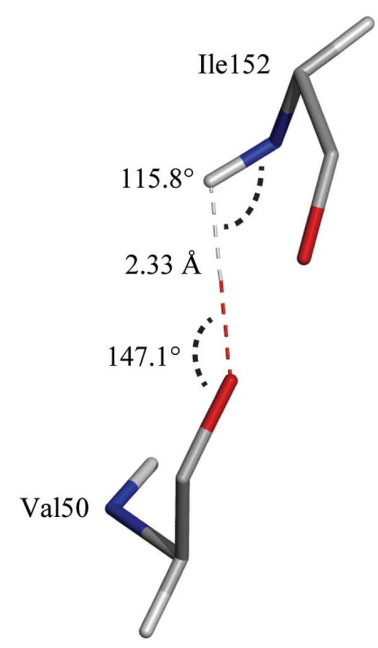

(c)

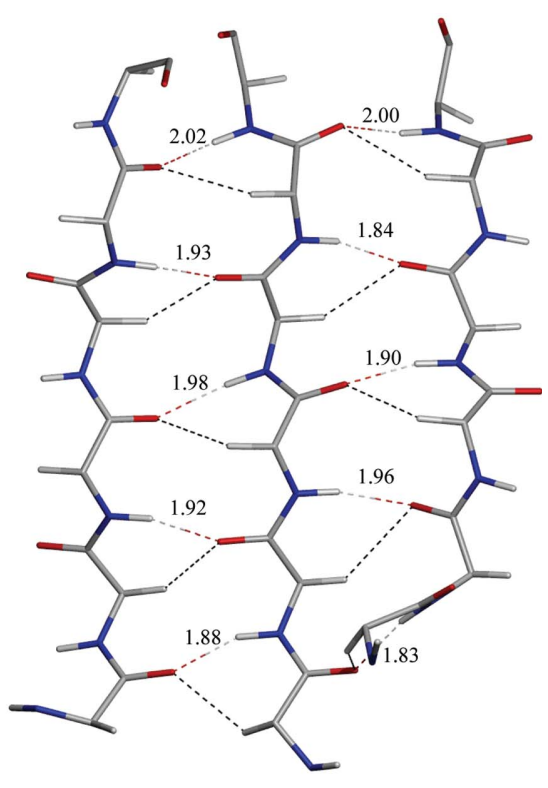

(b)

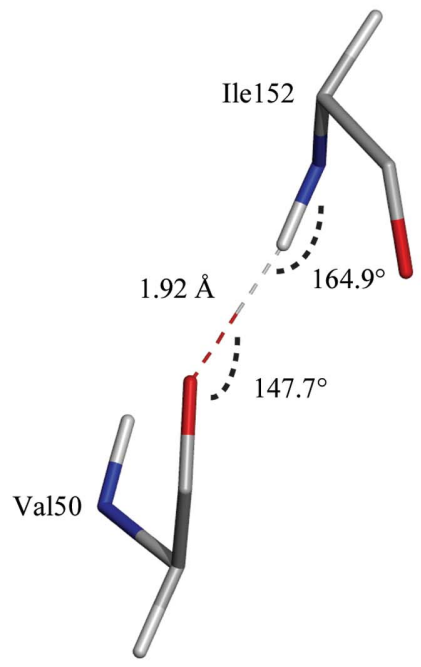

(d)
Figure 5

Three-stranded $\beta$-sheet domain in 3crw (4.0 ̊ resolution). (a) Refinement results using PHENIX and the automatic weighting scheme. (b) Refinement using $F F X$ and a weight of 1.0. $\beta$-Sheet hydrogen bonds are shown as dashed lines, with $\mathrm{N}-\mathrm{H} \cdots \mathrm{O}$ hydrogen bonds shown in red and white with distances in $\AA$ provided above them. Black dashed lines refer to 'weak' $\mathrm{C}^{\alpha}-\mathrm{H} \cdots \mathrm{O}$ hydrogen bonds that are also an important factor in $\beta$-sheet structure (Ho \& Curmi, 2002). (c) Detail of one hydrogen bond showing angle and distance values from PHENIX refinement. $(d)$ Detail of the same hydrogen bond as in $(c)$ with values from $F F X$-based refinement. quantum-mechanical potential energy surface in vacuum and, via polarization, experimental condensed phase properties including densities and heats of vaporization (Ren \& Ponder, 2003, 2004; Jiao et al., 2008; Ponder et al., 2010; Ren et al., 2011). On the other hand, it is not at all clear that the energetic sum of bond and angle restraints coupled to an Engh and Huber van der Waals nonbonded term, which has been further augmented by additional ad hoc restraints, is able to replicate the quantum-mechanical potential energy surface or any other well defined target. As an example, protein hydrogen bonds commonly occur through the free electron pairs of carbonyl $\mathrm{O}$ atoms, which have a nonlinear angle and distance dependence with respect to the hydrogen-bond donor (Baker \& Hubbard, 1984; Fabiola et al., 1997). This is illustrated in Fig. 5, where the FFX model properly captures the expected angle/ distance for both interacting lone-pair electrons (Fig. $5 d$ ), primarily as a result of the quadrupole description of the electrostatic potential around the carbonyl $\mathrm{O}$ atom. This bifurcated hydrogen bonding is an essential component in recapitulating twist and shear effects in $\beta$-sheet structures (Ho \& Curmi, 2002). Standard PHENIX refinement without electrostatics (Fig. 5c), atom-centered fixed-charge force fields and spring-based restraints (e.g. hydrogen-bond restraints; Echols et al., 2010) cannot capture the well defined angular dependence of this effect (Morozov et al., 2004) and therefore depends completely on the experimental data to provide this information. At low resolution, however, this information is weak or absent.

As hardware and software improve to enable the collection of data sets from crystals spanning an increasingly wider resolution range over increasingly complex conditions, the description and weighting of the chemical target term must be robust and transferable across varying environments to precisely recapitulate the atomic positions and forces that are present in the crystal. The limitations and difficulties of current methods and weighting schemes in dealing with these issues has been touched upon in the past (Jack \& Levitt, 1978; Lebedev et al., 2003; McCoy, 2004; Afonine et al., 2005a; Read, 2010). We suggest that the combination of a modern polarizable force field with a statically weighted maximum-likelihood crystallographic target yields robust state-of-the-art biomolecular models 
with increased information content. Furthermore, this approach alleviates the crystallographer from the burden of choosing the weighting factor manually and serves as a general approach for crystallographic model refinement.

\section{References}

Adams, P. D. et al. (2010). Acta Cryst. D66, 213-221.

Adams, P. D., Pannu, N. S., Read, R. J. \& Brünger, A. T. (1997). Proc. Natl. Acad. Sci. USA, 94, 5018-5023.

Afonine, P. V., Grosse-Kunstleve, R. W. \& Adams, P. D. (2005a). CCP4 Newsl. Protein Crystallogr. 42, 43-49.

Afonine, P. V., Grosse-Kunstleve, R. W. \& Adams, P. D. (2005b). Acta Cryst. D61, 850-855.

Arendall, W. B. III, Tempel, W., Richardson, J. S., Zhou, W., Wang, S., Davis, I. W., Liu, Z.-J., Rose, J. P., Carson, W. M., Luo, M., Richardson, D. C. \& Wang, B.-C. (2005). J. Struct. Funct. Genomics, 6, 1-11.

Baker, E. N. \& Hubbard, R. E. (1984). Prog. Biophys. Mol. Biol. 44, 97-179.

Berkholz, D. S., Shapovalov, M. V., Dunbrack, R. L. \& Karplus, P. A. (2009). Structure, 17, 1316-1325.

Bhattacharya, A., Tejero, R. \& Montelione, G. T. (2007). Proteins, 66, 778-795.

Bricogne, G. (2006). International Tables for Crystallography, Vol. B, Reciprocal Space, 1st online ed., edited by U. Shmueli, pp. 25-98. Chester: International Union of Crystallography.

Brünger, A. T. (1988). J. Mol. Biol. 203, 803-816.

Brünger, A. T. (1989). Acta Cryst. A45, 42-50.

Brünger, A. T. (1992). Nature (London), 355, 472-475.

Chen, V. B., Arendall, W. B., Headd, J. J., Keedy, D. A., Immormino, R. M., Kapral, G. J., Murray, L. W., Richardson, J. S. \& Richardson, D. C. (2010). Acta Cryst. D66, 12-21.

Cowtan, K. (2002). J. Appl. Cryst. 35, 655-663.

Cowtan, K. (2005). J. Appl. Cryst. 38, 193-198.

Darden, T., York, D. \& Pedersen, L. (1993). J. Chem. Phys. 98, 1008910092.

Echols, N., Headd, J. J., Afonine, P. \& Adams, P. D. (2010). Comput. Crystallogr. Newsl. 1, 12-17.

Engh, R. A. \& Huber, R. (1991). Acta Cryst. A47, 392-400.

Essmann, U., Perera, L., Berkowitz, M. L., Darden, T., Lee, H. \& Pedersen, L. G. (1995). J. Chem. Phys. 103, 8577-8593.

Fabiola, F., Bertram, R., Korostelev, A. \& Chapman, M. S. (2002). Protein Sci. 11, 1415-1423.

Fabiola, G. F., Krishnaswamy, S., Nagarajan, V. \& Pattabhi, V. (1997). Acta Cryst. D53, 316-320.

Faver, J. C., Benson, M. L., He, X., Roberts, B. P., Wang, B., Marshall, M. S., Sherrill, C. D. \& Merz, K. M. Jr (2011). PLoS One, 6, e18868.

Fenn, T. D., Schnieders, M. J. \& Brunger, A. T. (2010). Acta Cryst. D66, 1024-1031.

Fenn, T. D., Schnieders, M. J., Brunger, A. T. \& Pande, V. S. (2010). Biophys. J. 98, 2984-2992.
Fenn, T. D., Schnieders, M. J., Mustyakimov, M., Wu, C., Langan, P., Pande, V. S. \& Brunger, A. T. (2011). Structure, 19, 523-533.

Ford, G. C. \& Rollett, J. S. (1970). Acta Cryst. A26, 162.

Habeck, M., Rieping, W. \& Nilges, M. (2006). Proc. Natl. Acad. Sci. USA, 103, 1756-1761.

Ho, B. K. \& Curmi, M. G. (2002). J. Mol. Biol. 317, 291-308.

Jack, A. \& Levitt, M. (1978). Acta Cryst. A34, 931-935.

Jiao, D., Golubkov, P. A., Darden, T. A. \& Ren, P. (2008). Proc. Natl. Acad. Sci. USA, 105, 6290-6295.

Jorgensen, W. L. \& Tirado-Rives, J. (1988). J. Am. Chem. Soc. 110, $1657-1666$.

Lebedev, A. A., Tickle, I. J., Laskowski, R. A. \& Moss, D. S. (2003). Acta Cryst. D59, 1557-1566.

Linge, J. P., Williams, M. A., Spronk, C. A., Bonvin, A. M. \& Nilges, M. (2003). Proteins, 50, 496-506.

Lopes, P. E., Roux, B. \& Mackerell, A. D. (2009). Theor. Chem. Acc. 124, 11-28.

McCoy, A. J. (2004). Acta Cryst. D60, 2169-2183.

Morozov, A. V., Kortemme, T., Tsemekhman, K. \& Baker, D. (2004). Proc. Natl. Acad. Sci. USA, 101, 6946-6951.

Murshudov, G. N., Skubák, P., Lebedev, A. A., Pannu, N. S., Steiner, R. A., Nicholls, R. A., Winn, M. D., Long, F. \& Vagin, A. A. (2011). Acta Cryst. D67, 355-367.

Murshudov, G. N., Vagin, A. A. \& Dodson, E. J. (1997). Acta Cryst. D53, 240-255.

Nozinovic, S., Fürtig, B., Jonker, H. R., Richter, C. \& Schwalbe, H. (2010). Nucleic Acids Res. 38, 683-694.

Panigrahi, S. K. \& Desiraju, G. R. (2007). J. Biosci. 32, 677-691.

Ponder, J. W. \& Case, D. A. (2003). Adv. Protein Chem. 66, 27-85.

Ponder, J. W., Wu, C., Ren, P., Pande, V. S., Chodera, J. D., Schnieders, M. J., Haque, I., Mobley, D. L., Lambrecht, D. S., DiStasio, R. A., Head-Gordon, M., Clark, G. N., Johnson, M. E. \& Head-Gordon, T. (2010). J. Phys. Chem. B, 114, 2549-2564.

Read, R. J. (2010). Structure, 18, 664-665.

Ren, P. \& Ponder, J. W. (2003). J. Phys. Chem. B, 107, 5933-5947.

Ren, P. \& Ponder, J. W. (2004). J. Phys. Chem. B, 108, 13427-13437.

Ren, P., Wu, C. \& Ponder, J. W. (2011). J. Chem. Theor. Comput., doi:10.1021/ct200304d.

Sagui, C., Pedersen, L. G. \& Darden, T. A. (2004). J. Chem. Phys. 120, $73-87$.

Schnieders, M. J., Fenn, T. D. \& Pande, V. S. (2011). J. Chem. Theor. Comput. 7, 1141-1156.

Schnieders, M. J., Fenn, T. D., Pande, V. S. \& Brunger, A. T. (2009). Acta Cryst. D65, 952-965.

Schröder, G. F., Levitt, M. \& Brunger, A. T. (2010). Nature (London), 464, 1218-1222.

Ten Eyck, L. F. (1973). Acta Cryst. A29, 183-191.

Ten Eyck, L. F. (1977). Acta Cryst. A33, 486-492.

Touw, W. G. \& Vriend, G. (2010). Acta Cryst. D66, 1341-1350.

Tronrud, D. E., Berkholz, D. S. \& Karplus, P. A. (2010). Acta Cryst. D66, 834-842.

Vagin, A. A., Steiner, R. A., Lebedev, A. A., Potterton, L., McNicholas, S., Long, F. \& Murshudov, G. N. (2004). Acta Cryst. D60, 2184-2195.

Wang, H. \& Stubbs, G. (1993). Acta Cryst. A49, 504-513.

Waser, J. (1955). Acta Cryst. 8, 595. 